\title{
Flaubert lecteur de Spencer
}

De l'éducation intellectuelle, morale et physique dans Bouvard et Pécuchet

Yvan Leclerc

\section{(2) OpenEdition}

Journals

Édition électronique

URL : http://journals.openedition.org/aes/288

DOI : 10.4000/aes.288

ISSN : 2258-093X

Éditeur

Laboratoire LISAA

\section{Référence électronique}

Yvan Leclerc, «Flaubert lecteur de Spencer », Arts et Savoirs [En ligne], 4 | 2014, mis en ligne le 15 mai 2014, consulté le 20 avril 2019. URL : http://journals.openedition.org/aes/288 ; DOI : 10.4000/aes.288

Ce document a été généré automatiquement le 20 avril 2019.

Centre de recherche LISAA (Littératures SAvoirs et Arts) 


\title{
Flaubert lecteur de Spencer
}

\author{
De l'éducation intellectuelle, morale et physique dans Bouvard et
} Pécuchet

\section{Yvan Leclerc}

1 Il reste peu de traces matérielles des lectures de Spencer par Flaubert: elles tiennent toutes sur une seule page. Le nom du philosophe anglais se trouve mentionné dans quatre lettres, à chaque fois très brièvement ; les dossiers de Bouvard et Pécuchet comportent une seule référence bibliographique : réduite au seul nom de l'auteur, cette référence passe dans un ajout en marge d'un plan pour le dernier chapitre du roman inachevé, celui qui traite de l'éducation. Et c'est tout. Rien dans les carnets, ni dans l'inventaire de sa bibliothèque, ni dans les registres de prêts des bibliothèques publiques, il est vrai très lacunaires; pas de notes documentaires conservées. Ces rares informations factuelles permettent tout juste d'avancer quelques certitudes et surtout de formuler des hypothèses sur Flaubert lecteur de Spencer. Comment un romancier lit-il un philosophe? Flaubert n'est pas un philosophe ; c'est un écrivain qui pense, il ne lit pas les philosophes pour philosopher à son tour mais pour faire œuvre de fiction. Or, sa fiction est informée par la philosophie, elle inclut la philosophie comme matériau romanesque exposé dans un chapitre de Bouvard et Pécuchet, et elle a donné à penser aux philosophes depuis Taine jusqu'à Rancière, en passant par Sartre (dont il est de bon ton de se débarrasser d'un haussement d'épaules chez la plupart des flaubertiens, mais L'Idiot de la famille reste un monument, pas seulement par son nombre de pages).

2 Les quatre lettres de Flaubert qui mentionnent le nom de Spencer sont datées de 1878 et de 1880 , c'est-à-dire des trois dernières années de la vie de Flaubert, alors qu'il est en train de rédiger Bouvard et Pécuchet. Nous les donnons d'entrée ${ }^{1}$ :

Les Positivistes français se vantent: ils ne sont pas positivistes! Ils tournent au matérialisme bête, au d'Holbach! Quelle différence entre eux et un H. Spencer! Voilà un homme, celui-là ! - De même qu'on était autrefois trop mathématicien, on va devenir trop physiologiste ? - Ces gaillards-là nient tout un côté de l'homme, le côté le plus fécond et le plus grand (lettre à Edma Roger des Genettes, [12 janvier 1878], p. 347). 
Lisez-vous les œuvres d'Herbert Spencer ? Voilà un homme celui-là ! - et un vrai positiviste. Chose rare en France quoi qu'on die. - L'Allemagne n'a rien à comparer à ce penseur ! (lettre à Edma Roger des Genettes, $1^{\mathrm{er}}$ septembre [1878], p. 426).

Le livre de Robin sur la même matière [l'éducation] m'a paru peu fort. Et à celui de Spencer, j'ai éprouvé la même désillusion. Néanmoins, je voudrais bien les relire ! Arrange-toi pour que le P[ère] Didon m'expédie ce qu'il a, le plus promptement possible, et remercie-le, d'avance (lettre à sa nièce Caroline, [ $1^{\mathrm{er}}$ février 1880], p. 803).

Ne pas oublier de m'envoyer chez Pilon avec le paquet de Spencer les nouveaux documents sur Schopenhauer, l'engueulade à Challemel-Lacour, etc. (lettre à Guy de Maupassant, [25-26 février 1880], p. 848).

Flaubert a pu découvrir le philosophe avant la date de la première lettre dans laquelle il est mentionné : l'exclamation « Voilà un homme, celui-là ! » permet en effet de supposer que le jugement général repose sur une connaissance bien établie. Les livres de Spencer ont été publiés en anglais à partir des années 1850 , et en français après la guerre, les Premiers principes paraissant en $1871^{2}$. Flaubert lisait l'anglais, et il a pu ne pas attendre la traduction. Pour se tenir au courant de la production anglaise, et lui parler de Spencer, il pouvait compter en outre sur une personne qui avait pour nom le prénom du philosophe, Herbert, Juliette Herbert, sa maîtresse anglaise. Leurs lettres ont disparu mais on peut légitimement supposer qu'entre l'écrivain et l'institutrice cultivée, il n'était pas seulement question de sentiments tendres. Renan a pu jouer également le rôle de médiateur, puisqu'on sait que les deux philosophes étaient en relation épistolaire au plus tard en $1865^{3}$. Enfin, il est vraisemblable que le nom de Spencer soit venu jusqu'à Flaubert bien avant qu'il en ait lu un seul livre, par la diffusion précoce et rapide de sa pensée, dès le début des années 1860 en France, à travers les articles et les débats qu'il a suscités. En 1873, Elme-Marie Caro parle à son propos de "philosophie du moment »4. Lecteur de philosophes, Flaubert ne pouvait l'ignorer.

4 En France, la première étude ${ }^{5}$ parait dans la Revue des Deux Mondes le 15 février 1864 : un article d'Auguste Laugel intitulé «Les études philosophiques en Angleterre. M. Herbert Spencer, First Principles, by Herbert Spencer, London $1862 »^{6}$. Ce compte rendu analytique et critique, de vingt-sept pages, paraît donc deux ans après la publication du livre en Angleterre, longtemps avant sa traduction en français. On ne sait pas si Flaubert en a eu une connaissance directe; on sait en revanche que, comme tous ses contemporains cultivés, il lisait la Revue des Deux Mondes, dont il possédait des exemplaires dans sa bibliothèque, d'après l'inventaire après décès ${ }^{7}$; on sait également qu'il suivait avec intérêt et beaucoup de distance critique ce périodique auquel collaborait son ami Maxime Du Camp, et qui avait publié des « éreintements » de Madame Bovary et de Salammbô.

5 La date de lecture effective serait donc moins importante que l'époque où Flaubert prend connaissance de la philosophie de Spencer, fût-ce de seconde main, par un article savant ou un écrit de vulgarisation, selon le mode d'accès au savoir qui caractérise Bouvard et Pécuchet. Une chronologie, même vague, permettrait de départager au moins deux critiques qui mentionnent Spencer à propos de Flaubert. John Greene voit l'influence de Spencer dès La Tentation de saint Antoine, puisqu'il lui semble que «la notion de l'Inconnaissable, en délimitant les domaines de la science et de la foi, avait déjà cautionné le dénouement $»^{9}$ de cette œuvre, écrite entre 1870 et 1872, alors que Raymond Queneau s'en tient aux dates données par la correspondance : «[...] entre La Tentation et Bouvard et Pécuchet, Flaubert a lu Spencer et Bouvard et Pécuchet se termine sur une conclusion "sceptique", au sens où scepticisme et sciences sont identiques. $»^{10}$ 
Continuons à exploiter les indices contenus dans les quatre lettres de notre mince corpus. Une seule fois, dans la lettre du $1^{\text {er }}$ février 1880 (la troisième), Flaubert se réfère à un livre précis, De l'éducation intellectuelle, morale et physique, traduit en 1878. L'ouvrage n'est pas nommé, mais il ne peut s'agir que de lui dans le contexte des lectures pour le chapitre $\mathrm{X}$ de Bouvard et Pécuchet. Nous avons affaire ici à une lecture documentaire ciblée, à finalité explicite. Dans les trois autres lettres, Spencer est mentionné comme un tout, dans sa globalité représentée singulièrement par son nom (« un H. Spencer !»), par l'ensemble de son œuvre («les œuvres d'Herbert Spencer») ou par une quantité indéterminée, «le paquet de Spencer » pouvant contenir un seul ou plusieurs volumes du philosophe, en plus d'autres études sur ses ouvrages. Il ne s'agit plus ici de lectures à finalité immédiate, mais de grandes lectures de fond qui convoquent non tel ou tel livre mais la pensée dans son amplitude. Flaubert lit « du » Spencer, avec l'exacte valeur du partitif grammatical : la partie d'un tout, la partie valant pour le tout, puisque le tout de la pensée se manifeste dans chacun des livres. Quand on a lu un livre de Spencer, on accède à tout Spencer.

Flaubert s'intéresse au système, à un principe d'unité et de cohérence. Certes, Bouvard et Pécuchet est un roman "anti-système" (comme dirait une partie de notre personnel politique) : les systèmes y sont tournés en farce dans leur prétention à universaliser une pensée partielle, à se donner comme le «meilleur système ", à s'affronter en systèmes contradictoires : «Et tous deux s'avouèrent qu'ils étaient las des philosophes. Tant de systèmes vous embrouille. $~^{11}$ Mais la condamnation de l'esprit de système laisse place chez Flaubert à la séduction pour les constructions totalisantes, pour ce qu'il appelle ailleurs, dans un autre contexte, la "grande synthèse ${ }^{12}$.

Chez Spencer, le système prend la forme d'une loi unique qui traverse les règnes et embrasse tous les domaines de la connaissance. Flaubert apprécie les entreprises à visée encyclopédique : tous ses romans sont, chacun à sa manière, des encyclopédies, et des encyclopédies critiques, sérieuses ou en farce. En ce sens, Spencer est son homme; Auguste Laugel signale d'emblée chez lui «la variété presque encyclopédique dont ses écrits fournissent la preuve $»^{13}$.

9 En progressant dans la lecture de l'article de Laugel, on voit se multiplier les points de contact entre la pensée de Spencer et les idées de Flaubert, telles qu'elles sont exposées dans sa correspondance. Laugel présente Spencer comme un héritier de la Philosophie positive de Comte, «[q]uoique non avouée», dit-il ${ }^{14}$. Mais quand il prit connaissance de l'article de Laugel, dont il loue par ailleurs l'exposé fait avec soin, sympathie et bonne foi, Spencer lui-même répondit dans un article intitulé «Pourquoi je me sépare d'Auguste Comte", publié dans la traduction française de Classification des sciences en $1872^{15}$. Il souligne d'abord les points communs de sa philosophie avec celle de Comte, en prenant soin de préciser que ce qui le rapproche de ce dernier se trouvait déjà dans la pensée de philosophes bien antérieurs à son aîné : l'expérience comme source de la connaissance, la relativité de la connaissance qui n'atteint que des phénomènes, le refus de recourir «à des entités métaphysiques pour expliquer ces phénomènes ${ }^{16}$. Spencer présente ensuite ce qui le distingue de Comte sous la forme de citations disposées sur deux colonnes en visà-vis: aux trois états de Comte, Spencer oppose une seule méthode; il inverse la subordination comtienne de la vie individuelle à la vie sociale; il conforte le sentiment religieux qui trouve son accomplissement dans l'« Infini Incognoscible» et non dans le « Fini Cognoscible ${ }^{17}$ de Comte qui achève son système dans une autolâtrie humanitaire et un culte de la vie collective devenant l'Être suprême. Même si Flaubert n'a pas eu connaissance de cette mise au point de Spencer après l'article de Laugel, il n'a pu que 
percevoir immédiatement les différences entre la philosophie exposée dans Premiers principes et la philosophie de Comte, qu'il avait condamnée depuis longtemps : «Il y a làdedans des mines de comique immenses, des Californies de grotesque. $»^{18}$ Ainsi, Flaubert marquera-t-il l'opposition entre le "vrai positiviste» qu'est Spencer et l'école comtienne : « Les Positivistes français se vantent : ils ne sont pas positivistes! Ils tournent au matérialisme bête, au d'Holbach! Quelle différence entre eux et un Spencer !»

Cette différence tient en plusieurs points. Comte propose un positivisme fermé, dogmatique, " conclusif», transformant la science et l'homme en objets absolus de culte et de croyance. La philosophie comtienne engendre des Homais. Flaubert a toujours eu mépris et sarcasmes pour les philosophies qui font de l'homme la mesure et la fin de toutes choses. En outre, Comte conçoit l'état positif comme un dépassement et une annulation des états théologiques et métaphysiques antérieurs, alors que pour Spencer comme pour Flaubert la religion, ou plutôt le « sentiment religieux » est « profondément enraciné dans le cœur de l'homme. ${ }^{19}$ L'auteur de La Tentation de saint Antoine parle du sentiment religieux comme d'un « sentiment poétique » :

[...] ce qui m'attire par-dessus tout, c'est la religion. Je veux dire toutes les religions, pas plus l'une que l'autre. Chaque dogme en particulier m'est répulsif, mais je considère le sentiment qui les a inventés comme le plus naturel et le plus poétique de l'humanité. Je n'aime point les philosophes qui n'ont vu là que jonglerie et sottise. J'y découvre, moi, nécessité et instinct; aussi je respecte le nègre baisant son fétiche autant que le catholique aux pieds du Sacré-Cœur. ${ }^{20}$

11 Il n'est pas impossible de lire comme un énoncé sérieux, par exception, ce passage du chapitre de l'éducation dans Bouvard et Pécuchet: « Mais le raisonnement ne satisfait pas tous les besoins. Le cœur et l'imagination veulent autre chose $»^{21}$. Au lieu d'opposer le matérialisme et le spiritualisme, et de postuler comme Comte que la science résorbera la part d'irrationnel en l'homme, Spencer donne à Flaubert l'exemple d'un philosophe qui les pense ensemble, dans une volonté de « réconciliation » et de fusion ${ }^{22}$, en les réunissant négativement par l'Inconnaissable (Laugel parle du " cognoscible » et de "l'incognoscible » ${ }^{23}$ ). Certaines phrases de Spencer, citées par Laugel, sont superposables à celles de Flaubert, par exemple quand le philosophe écrit :

[...] les raisonnements contenus dans ces pages ne prêtent d'appui à aucune des hypothèses antagonistiques relatives à la nature dernière des choses. Ils ne sont pas plus favorables au matérialisme qu'au spiritualisme, au spiritualisme qu'au matérialisme. Tout argument qui semble favorable à l'une de ces deux causes est neutralisé par un aussi bon argument fourni à la cause contraire. ${ }^{24}$

Spencer maintient une tension égale entre les deux conceptions, là où Flaubert les renvoie dos à dos, mais de part et d'autre la volonté est égale de ne pas les séparer. «Je trouve le Matérialisme et le Spiritualisme deux impertinences égales ». À quelques variantes près, on retrouve cette phrase à trois reprises dans la correspondance ${ }^{25}$. Dans sa thèse sur L'Âme et le corps chez Flaubert, Juliette Azoulai remarque fort justement que le terme " impertinence » est à comprendre en deux sens, à la fois logique et moral : ce qui n'est pas pertinent sur le plan de la vérité, et ce qui est impertinent parce que péremptoire ${ }^{26}$. On pourrait ainsi multiplier les flaubertismes de Spencer ou les spencérismes de Flaubert, dans l'ordre de la pensée et de l'expression.

Eugène Laugel conclut son étude par la remarque suivante : « Il nous a paru qu'il y avait quelque chose d'original et je dirais volontiers de poétique dans cette forme nouvelle sous laquelle apparait aujourd'hui une doctrine qu'on a quelquefois, et à bon droit, pu accuser de sécheresse et de dureté. ${ }^{27}$ En quoi la doctrine de Spencer peut-elle apparaître 
poétique à Laugel ? Probablement en ce qu'elle réalise philosophiquement le vieux rêve romantique de l'unité primordiale, à travers la dynamique d'une force. Du point de vue de Flaubert, la poésie naît aussi de la conception spencérienne que la pensée ou « l'énergie mentale n'est qu'une métamorphose de la force vitale $! »^{28}$. Or la métamorphose est plus qu'un thème poétique; c'est la matrice même de l'imaginaire poétique, depuis la mythologie jusqu'à l'œuvre de Flaubert, en particulier dans La Tentation de saint Antoine, et même dans Bouvard et Pécuchet : le changement constant des formes, la ductilité et la fluidité de la matière rendant impossibles la nomination et la fixité du savoir. Dans leur introduction au volume Penser Spencer, beau titre presque anagrammatique, Daniel Becquemont et Dominique Ottavi soulignent la nécessité de s'intéresser aussi «à l'action d'un imaginaire scientifique $»^{29}$. Cet imaginaire est d'autant plus prégnant quand le lecteur est un écrivain. Flaubert lit Spencer pour ses idées, mais il le lit également littéralement, en tant que romancier sensible aux formes de l'expression, à ce qu'on pourrait appeler, en empruntant l'expression à la rhétorique mais en lui donnant un sens philosophique, des figures de pensée.

Comment dès lors expliquer que Flaubert, admiratif de Spencer en général, émette des réserves sur le seul livre qu'il mentionne sinon par son titre, du moins par son sujet, l'essai sur l'éducation ? Flaubert le connaissait déjà, puisqu'il en parle au passé, à l'époque où il commence à rédiger le chapitre $\mathrm{X}$ et dernier de Bouvard et Pécuchet, portant sur l'éducation: «Le livre de Robin sur la même matière m'a paru peu fort. Et à celui de Spencer, j'ai éprouvé la même désillusion. Néanmoins, je voudrais bien les relire ! »

Flaubert a pu lire De l'éducation intellectuelle, morale et physique deux ans plus tôt, la traduction française ayant paru en 1878. Dans les dossiers de Bouvard et Pécuchet se trouve une fiche bibliographique sur laquelle est inscrite la référence à cet ouvrage. La fiche n'est pas de la main de Flaubert ${ }^{30}$ :

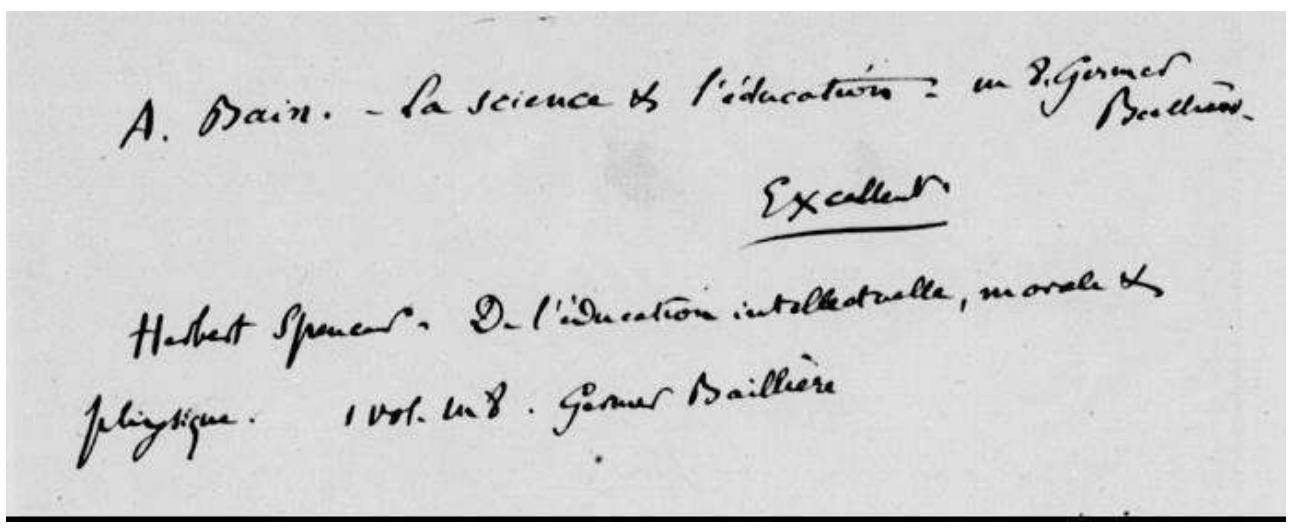

Quelques lignes d'envoi en tête de ce folio, mentionnant la présence de ces livres au muséum, font penser que la fiche est écrite, ou du moins envoyée par Georges Pouchet, directeur du Muséum d'histoire naturelle de Rouen, à qui Flaubert avait demandé de la documentation ${ }^{31}$. C'est parce qu'il ne répond pas immédiatement que Flaubert presse sa nièce de solliciter le Père Didon, son confesseur. Pour avoir des livres sur l'éducation, on peut s'adresser à un scientifique ou à un théologien, ce qui situe bien le champ de la discipline et celui qu'occupe Spencer.

Sur la liste bibliographique, entre les références aux deux traités sur l'éducation de Bain et de Spencer, on lit cette appréciation de la main de Flaubert, semble-t-il, et soulignée : "Excellent». Le jugement se rapporte très probablement au livre de Bain, puisque Flaubert trouve celui de Spencer rien moins qu'excellent. A-t-il eu le temps de le relire 
quand il l'a reçu, quinze jours après la lettre à sa nièce ? En tout cas, le nom de Spencer ne figure pas dans la liste des 43 références que Flaubert a établie pour regrouper les ouvrages utilisés dans la rédaction du chapitre $\mathrm{X}$, sous le titre «Morale, Éducation, Phrénologie, Administration $»^{32}$.

Le nom de Spencer ne se rencontre qu'une seule fois dans le dossier génétique du roman, dans un encadré figurant en haut d'un plan du chapitre X. On lit : « Stuart Mill/Spencer/ Bain, etc. $»^{33}$

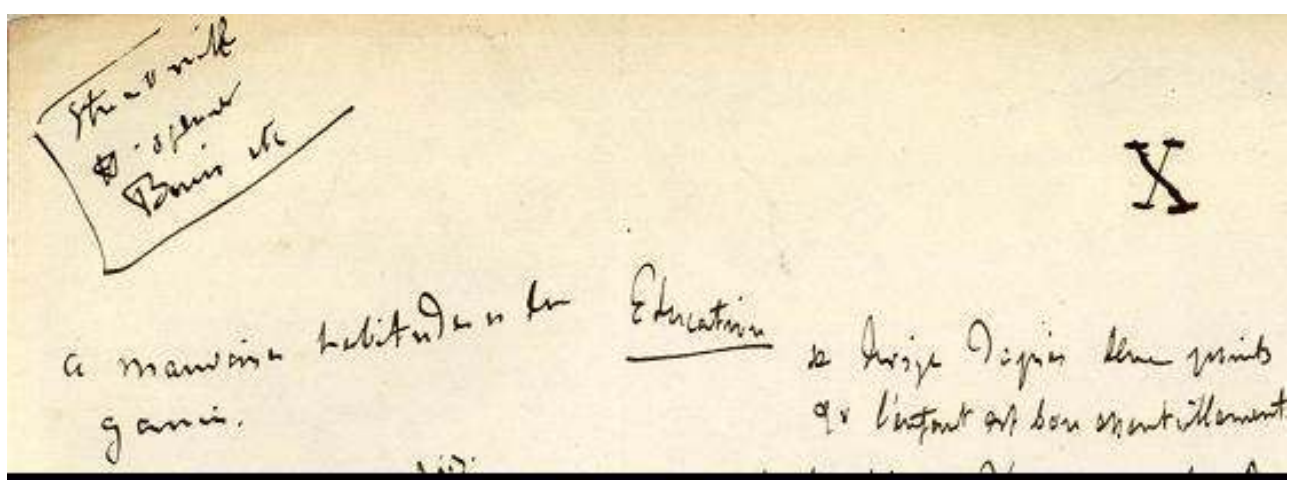

Spencer n'apparaît plus ensuite, non seulement dans le texte définitif, mais même dans les brouillons, pourtant beaucoup plus riches en matière et en noms propres que le dernier état laissé par Flaubert. Pourquoi ce silence dans le texte, après un jugement défavorable dans une lettre? Les deux n'étant pas liés d'ailleurs, et même au contraire, puisque Bouvard et Pécuchet est fait surtout de livres que Flaubert juge " peu forts ", pour reprendre l'expression dont il use à propos du traité de Spencer sur l'éducation. Tout au plus pourrait-on supposer que Flaubert, admirant la pensée de Spencer, a évité de le faire apparaître à son désavantage en mentionnant un livre qui fait exception. À ce jugement négatif et à cette absence, on pourrait trouver quatre explications.

\section{Première explication}

Ce que Flaubert pourrait retenir de "l'art d'élever les enfants", tel que le conçoit Spencer, il l'a déjà trouvé ailleurs, et en particulier dans l'Émile, qui est la référence majeure du chapitre $\mathrm{X}^{34}$. Le nom ou plutôt le prénom de «Jean-Jacques », et le titre Émile ne sont mentionnés qu'une seule fois dans ce chapitre, mais la lecture des notes prises par Flaubert et l'étude des manuscrits montrent que le texte rousseauiste fournit la matière d'une demi-douzaine de citations sans origine disséminées dans l'épisode. Spencer ne mentionne jamais Rousseau dans son traité, pour les raisons données par Dominique Ottavi : Spencer s'intéresse à la pratique de l'éducation et non à la théorie, alors que Flaubert s'intéresse aux deux, et surtout aux contradictions entre les deux. On va donc trouver dans Bouvard et Pécuchet Rousseau et Pestalozzi, alors que Spencer ne cite que Pestalozzi, le pédagogue suisse disciple de Rousseau, dont il approuve la doctrine, tout en émettant des réserves sur ses applications pratiques : « Nous sommes donc ainsi mis sur la voie de la doctrine depuis longtemps proclamée par Pestalozzi, à savoir que, dans son ordre comme dans ses méthodes, l'éducation doit se conformer à la marche naturelle de l'évolution mentale. $»^{35}$ Pestalozzi est cité dans le roman: "Pécuchet objecta que les châtiments corporels sont quelquefois indispensables. Pestalozzi les employait [...]. »" Même si Spencer ne fait pas l'objet d'une mention, il est clair qu'il est inclus implicitement dans le système que les deux pédagogues adoptent, à l'incipit du chapitre : 
Ils se procurèrent plusieurs ouvrages touchant l'éducation - et leur système fut résolu. Il fallait bannir toute idée métaphysique, - et d'après la méthode expérimentale suivre le développement de la nature. Rien ne pressait, les deux élèves devant oublier ce qu'ils avaient appris. ${ }^{37}$

La "méthode expérimentale ", le "développement de la nature », le passage par une phase pédagogique négative pendant laquelle il faut faire table rase des savoirs précédemment acquis, sont communs à Rousseau et à Spencer, de même que, dans le traité du philosophe anglais, la confiance accordée à une «éducation spontanée » et aux " instincts naturels ${ }^{38}$, un enseignement fondé sur la liberté et sur le plaisir plutôt que sur la contrainte ${ }^{39}$, la démarche anti-dogmatique qui consiste à partir du concret pour aller vers l'abstrait, du particulier vers le général et de l'empirique vers le rationnel. Spencer prend l'exemple de la grammaire, abstraction de la langue et rationalisation de son fonctionnement, par laquelle on ne saurait commencer un enseignement: il faut apprendre à parler avant de théoriser le discours ${ }^{40}$. L'enseignement de la morale doit être lui aussi «naturel », en ce sens, chez les deux philosophes, que la punition doit être pour ainsi dire immanente à la faute, sa conséquence directe, ce que Spencer appelle la « réaction naturelle $»^{41}$. La méthode «naturelle» de l'un et de l'autre se fonde sur une condamnation de l'enseignement par les livres, au moins dans un premier temps: Spencer entend « soustraire les enfants à la routine des livres » et condamne la récitation " par cœur ", la " vieille méthode mécanique », dit-il. La « leçon de choses " prend le pas sur la leçon de vocabulaire: «Apprendre le sens des choses vaut mieux qu'apprendre le sens des mots $»^{42}$.

Sur tous ces points de convergence, Spencer apparaît surnuméraire et répétitif par rapport à la pédagogie de Rousseau, tellement popularisée auprès du public français qu'il n'est pas nécessaire de la redoubler en faisant référence au traité du philosophe anglais.

\section{Deuxième explication}

Une autre raison peut expliquer l'absence de la référence explicite à Spencer dans Bouvard et Pécuchet, inverse de la précédente, aboutit cependant au même silence. Flaubert partage les vues de Spencer, cette fois contre les conceptions de Rousseau, mais les convictions du romancier sont tellement fortes, elles sont ancrées en lui depuis si longtemps qu'il peut se passer d'une caution extérieure. Deux exemples viennent immédiatement à l'esprit.

Contre Rousseau, Spencer prône l'égalité des filles et des garçons devant l'enseignement. Il s'indigne en effet que l'on cantonne les filles dans des apprentissages superflus et décoratifs, et que, par exemple, on ne leur fasse pas pratiquer d'exercices physiques. Certaines paroles de la correspondance ont pu faire accuser Flaubert de misogynie, mais dans le domaine de l'éducation, il ne semble pas avoir marqué de différence entre les sexes, par exemple dans l'enseignement qu'il dispensait lui-même à sa nièce. Ce point de l'éducation spécifique suivant le sexe fait débat entre Bouvard et Pécuchet, sans que l'on sache bien, dans le brouillage des voix de la première citation, qui défend quoi :

Maintenant qu'ils [les deux enfants] savaient écrire et lire, que leur apprendre?

Autre embarras. Les filles n'ont pas besoin d'être savantes comme les garçons. N'importe! On les élève ordinairement en véritables brutes, tout leur bagage se bornant à des sottises mystiques. ${ }^{43}$ 
[...] il est cruel, objecta Pécuchet, d'élever les filles en vue exclusivement du mari qu'elles auront. Toutes ne sont pas destinées à l'hymen. Et si on veut que plus tard elles se passent des hommes, il faut leur apprendre bien des choses. ${ }^{44}$ hugolienne, à Jean Valjean et au prénom de l'auteur, puisque les enfants s'appellent Victor et Victorine. Les Misérables est un plaidoyer pour le progrès par l'éducation; à l'inverse, Bouvard et Pécuchet montre qu'elle ne peut rien sur deux enfants à l'hérédité chargée, hantés par des images traumatisantes. La phrénologie, pseudo-science dans laquelle Bouvard et Pécuchet réussissent, confirme ces prédispositions, puisque le « crâne déplorable» de Victor indique "l'organe de la destruction, du meurtre; [...] de la convoitise, du vol $\aleph^{48}$. Effectivement, Victor réalise le programme génétique moulé sur son crâne en actualisant, en mineur, tous les vices inscrits dans les proéminences de sa boîte crânienne $:$ il vole, il donne " une violente raclée " ${ }^{49}$ à un autre enfant, il détruit la noix de coco de Pécuchet; et, avec sa sœur, il fait bouillir un chat dans une marmite. «Ensuite Bouvard et Pécuchet causèrent longuement de Victor. Le sang paternel se manifestait. Que faire ? [...] - "La sœur ne vaut pas mieux", dit Bouvard. $»^{50}$ Flaubert rejoint ici Spencer quand il renverse l'idée reçue de l'innocence des enfants (les enfants ne sont pas " nés bons ${ }^{51}$ ), leurs «mauvais instincts» naturels étant aggravés par l'hérédité des caractères acquis : «Si la transmission héréditaire est une loi de la nature, [...] alors, dans la moyenne des cas, les défauts des enfants sont le reflet des défauts des parents $\aleph^{52}$. Donc, Spencer a raison, mais Flaubert en est si convaincu d'avance qu'il n'a pas besoin de s'y référer.

L'éducation «naturelle» ne peut qu'atténuer cette double imperfection elle aussi naturelle, individuelle et familiale, et non l'éliminer. Chez Spencer, l'éducation se loge dans la marge du «presque tout » de Flaubert: la Nature ne fait pas tout. Encore faut-il 
appliquer aux enfants une bonne méthode. Ce qui, évidemment, fait question dans Bouvard et Pécuchet, quand les deux pédagogues concluent :

- «Hélas », reprit Pécuchet. «Mais il y a des natures dénuées de sens moral; - et

l'éducation n'y peut rien.»

- «Ah! oui ! c'est beau, l'éducation. $»^{53}$

Est-ce une conclusion spencérienne? Oui, dans le constat qu'il existe des natures immorales, mais non pour la défaite complète de l'éducation. La thèse que Flaubert voulait démontrer ne résiste guère à sa mise en fiction : «l'Éducation, quelle qu'elle soit, ne signifie pas grand-chose ». La thèse aurait été probante si Flaubert avait écrit, après Spencer, un traité d'éducation. Mais il écrit un roman avec des personnages qui se prennent pour des pédagogues et qui expérimentent des méthodes contraires : il n'est pas seulement question d'éducation, mais d'éducateurs, pas seulement de théorie, mais d'applications pratiques. Et Bouvard et Pécuchet tomberaient sous la critique adressée par Spencer au disciple suisse de Rousseau: comme Pestalozzi, les deux bonshommes appliquent mal de bonnes idées.

\section{Troisième explication}

31 La troisième raison de l'absence de Spencer nous rapproche de la « désillusion » éprouvée par Flaubert à la lecture de son livre sur l'éducation. On sait que le romancier place très haut la science, qu'il en fait un modèle et une méthode, que dans Bouvard et Pécuchet il ne critique pas la science mais les savoirs ${ }^{54}$, qu'il rêve d'une " littérature [...] qui prendra de plus en plus les allures de la science " et d'un style "précis comme le langage des sciences $»^{55}$. Ces citations sont bien connues. Mais jamais Flaubert ne subordonne la Littérature (qu'il écrit souvent avec une majuscule) à la science. La littérature ne doit pas disparaitre dans la science, c'est au contraire la science qui doit servir la littérature. Sur cette question du rapport entre la science et les lettres, la position de Spencer est différente. D'une part, il montre la poésie de la science, qui ouvre aux savants des mondes imaginaires, en particulier la géologie, dévoilant « le grand poème épique que le doigt de Dieu a écrit sur les couches de la terre $! »^{56}$. À la métaphore hyperbolique près, qui aurait pu entraîner cette phrase dans la rubrique «Beautés de la science » du second volume de Bouvard et Pécuchet, Flaubert partage cet enthousiasme.

Mais il se sépare radicalement du philosophe quand celui-ci oppose la poésie naturelle des sciences à la poésie « artificielle » et à plusieurs disciplines qui appartiennent à ce qu'on appelle aujourd'hui les sciences humaines. En lisant le premier chapitre du traité sur l'éducation, "Quel est le savoir le plus utile», on mesure en effet la désillusion de l'écrivain Flaubert, puisque le philosophe élimine du programme le latin et le grec qui "neuf fois sur dix sont inutiles à un jeune homme", l'étude des langues qui ne fait pas appel à la raison, et l'histoire qui «n'a pas la plus légère influence sur nos actions $»^{57}$. La disqualification de ces disciplines s'explique par le but que Spencer assigne à l'éducation : l'utilité, et par l'idée qu'il se fait des futurs bénéficiaires de son système pédagogique : au fil des pages sont nommés un boutiquier, un employé de bureau, un propriétaire, une mère et un père de famille, un directeur de banque ainsi que - coup de grâce pour Flaubert -, le directeur d'un chemin de fer et un conducteur de locomotive... Il est vrai que Spencer a fait ses débuts dans les chemins de fer. Si l'on traduit tous ces métiers et statuts sociaux dans le vocabulaire flaubertien, ce sont des bourgeois, ceux qui défendent les «bases » de la société, telles qu'elles sont définies dans le Dictionnaire des idées reçues: 
la propriété et la famille. On pourrait ainsi multiplier les énoncés spencériens qui vont dans le sens d'une condamnation de la "culture esthétique» considérée comme "subsidiaire $~_{58}$. Certaines phrases de l'ouvrage semblent écrites contre le credo flaubertien, par exemple: "ce qui forme le citoyen doit passer avant ce qui forme l'artiste »; " on ne peut pas dire que l'élégance et la correction du style soient choses aussi utiles à posséder que les principes qui peuvent nous conduire à élever nos enfants » ${ }^{59}$, etc.

Ainsi, la désillusion de Flaubert tient-elle moins à la méthode qu'au mépris affiché par le philosophe-pédagogue pour ces valeurs de luxe social qui lui sont si nécessaires (l'histoire, les langues, l'art, le style), et qu'à l'horizon social de l'enseignement spencérien, destiné à former de bons citoyens et de bons chefs de famille.

\section{Quatrième explication}

Aux prises avec des matières de plus en plus abstraites dans son roman, Flaubert cherche dans les discours des savoirs ce qui peut donner lieu à la mise en fiction, sous forme de dialogues, de micro-récits, de descriptions, de saillies comiques ${ }^{60}$. Il s'agit donc de rendre les idées " plastiques ", de leur donner forme narrative, dialoguée, descriptive. Or, le livre de Spencer ne fournit pas de bonne matière plastique. C'est un livre sans effet, neutre, sans images, sans métaphores, avec quelques exemples vécus pour illustrer la méthode pédagogique, mais sans anecdote relevée. La « figurabilité » du savoir y est inexistante. Si bien que Flaubert emprunte ailleurs ses exemples. Il trouve chez Spencer, comme dans Rousseau et Bentham ${ }^{61}$, la théorie des "réactions naturelles " "2 , qui consiste, on l'a vu, à faire supporter par les enfants les conséquences inévitables des actes qu'ils commettent. Mais c'est dans l'Émile que Flaubert trouve un exemple simple et frappant. Rousseau écrit : « Il casse les fenêtres de sa chambre ; laissez le vent souffler sur lui nuit et jour sans vous soucier des rhumes. $»^{63} \mathrm{Ce}$ qui devient dans la syntaxe courte et syncopée de Bouvard et Pécuchet : «L'enfant a brisé un carreau, on n'en remettra pas, qu'il souffre du froid. $»^{64}$ Pour sa part, Spencer donne d'autres exemples : un enfant a laissé traîner ses jouets sur le plancher et il a refusé de les ramasser ; ses parents le punissent en le privant de sa boîte à jouets la fois suivante ${ }^{65}$. Mais de telles illustrations sont si plates, si banales qu'elles laissent sans prise le romancier à la recherche de détails ou de situations susceptibles de se transformer en micro-séquences narratives.

Si donc le livre de Spencer provoque la désillusion, c'est parce que Flaubert n'y apprend rien qu'il n'ait lu ailleurs ou qu'il ne pense lui-même fortement; que Spencer, tout en condamnant l'« utilitarisme grossier " ${ }^{66}$, ne conçoit qu'un enseignement utile pour former des bourgeois et que son livre écrit contre les mots subit le châtiment immanent et mérité de ne pas être écrit.

Il est difficile de savoir comment un écrivain lit un autre écrivain ; encore plus difficile de savoir comment un écrivain lit un philosophe. Claude Digeon, l'un des rares flaubertiens à avoir parlé de Spencer d'une manière informée dans un livre déjà ancien, Le Dernier Visage de Flaubert, écrit: "Nous avons le droit de supposer qu'il a trouvé chez ce philosophe, sous une forme proprement intellectuelle, ce que lui-même pensait depuis longtemps déjà de façon plus confuse et plus impulsive. ${ }^{{ }^{67}}$ Cette hypothèse est discutable: le philosophe n'apporte pas la clarté ou la réflexion là où il n'y avait que confusion et impulsion, d'autant plus que Flaubert lit Spencer à l'âge mûr. Mais nous serions en peine de proposer une meilleure analyse du processus romanesque déclenché par la lecture de 
Spencer. Peut-être y a-t-il eu reconnaissance, au double sens où le lecteur Flaubert reconnaît ses propres conceptions (il s'y retrouve), en même temps qu'il éprouve de la gratitude à l'égard d'un penseur qui lui correspond si bien. Au fond, Flaubert était spencérien avant d'avoir lu Spencer; Spencer ne lui a pas révélé un monde, mais il l'a conforté dans le sien.

Revenons pour finir à notre hypothèse initiale : Flaubert découvre probablement Spencer, comme de nombreuses personnes cultivées de son temps, par l'article inaugural d'Auguste Laugel dans la Revue des Deux Mondes, en 1864. Au tout début, le critique donne une indication biographique qui n'a pas dû manquer de retenir l'attention de notre lecteur :

[...] M. Spencer, s'il eût consenti à suivre les routes battues, aurait facilement obtenu tous ces dons que la société anglaise prodigue à ceux qui la servent comme elle veut être servie. Il a préféré se condamner à la pauvreté, et, ce qui est encore plus difficile, à l'obscurité. Cette abnégation a quelque chose de fier et de touchant ; mais M. Spencer mérite mieux que de vaines marques de sympathie : il ne faut pas seulement admirer sa fidélité à des études sans profit ; son œuvre mérite par ellemême d'attirer l'attention de tous les amis de la philosophie. ${ }^{68}$

Certes, l'œuvre importe plus que l'homme, et ce n'est pas Flaubert, théoricien de l'impersonnalité, qui contredira Laugel. Mais cette information biographique prend toute sa valeur précisément en rapport avec l'œuvre, puisque la pauvreté, l'obscurité, le refus des honneurs, des grades et des titres, des distinctions académiques est en cohérence avec les principes éthiques et politiques du philosophe anglais. En tant que libéral conséquent, il ne demande rien à l'État, ni protection, ni aide, ni pension. Flaubert admire ceux qui vivent selon des principes clairs régissant à la fois leur art et la conduite de la vie, même si lui-même a eu la faiblesse parfois de déroger. On mesure mieux son humiliation au moment où, ruiné par sa nièce, il se voit dans l'obligation d'accepter une pension de l'État. Ce n'est pas seulement l'individu Flaubert qui est en jeu, mais au-delà de son cas personnel, la conception qu'il se fait, avec Spencer, des rapports entre l'individu et la société. L'autonomie de l'art par rapport aux valeurs non esthétiques doit trouver son corollaire dans l'indépendance du créateur vis-à-vis de la société, dans une mise en conformité de la vie et de la pensée. Ce n'est peut-être pas par hasard que dans les premières lettres où il cite Spencer, à quelques mois de distance, Flaubert utilise la même expression louangeuse : «Voilà un homme, celui-là !» Un homme, à propos de Spencer, c'est plus et autre chose qu'un philosophe. Certes, Flaubert pourrait dire la même chose d'Homère, dont on ne connaît pas la biographie. Mais pour Spencer, c'est différent : le statut de pauvreté fait partie intrinsèque de sa pensée. L'homme Spencer que Flaubert admire, c'est la philosophie faite homme, la preuve éthique de la valeur d'un système, la traduction d'une philosophie dans une vie.

\section{NOTES}

1. Les quatre citations renvoient à Flaubert, Correspondance, cinq tomes, éd. Jean Bruneau et Yvan Leclerc, Paris, Gallimard, t. V, 2007, coll. « Bibliothèque de la Pléiade ». 
2. Les ouvrages de Spencer, Premiers principes, Classification des sciences, Principes de psychologie, Essais de morale, de science et d'esthétique et De l'éducation intellectuelle, morale et physique se trouvent à la Bibliothèque municipale de Rouen (aujourd'hui dans le fonds ancien), où Flaubert a pu les emprunter.

3. Patrick Tort, Spencer et l'évolutionnisme philosophique, Paris, PUF, 1996, p. 33, coll. «Que saisje?».

4. Voir Dominique Ottavi, «Spencer et la France », dans Penser Spencer, sous la direction de Daniel Becquemont et Dominique Ottavi, Saint-Denis, Presses Universitaires de Vincennes, 2011, p. 13-24, ici p. 18, coll. « La philosophie hors de soi ».

5. Selon Ottavi, ibid., p. 17. Toujours selon la même source, cet article est signalé dans la préface de la traduction des Premiers principes, trad. par M. E. Cazelles, Paris, G. Baillière, 1871.

6. Auguste Laugel, « Les études philosophiques en Angleterre. M. Herbert Spencer, First Principles, by Herbert Spencer, London 1862 », Revue des Deux Mondes, 15 février 1864, p. 930-957.

7. L'inventaire des biens de Flaubert après son décès montre qu'il avait huit volumes de la Revue des Deux Mondes (non conservés) dans sa bibliothèque, mais la date n'est pas indiquée. Voir l'inventaire transcrit par Matthieu Desportes sur le site Flaubert : http://flaubert.univ-rouen.fr/ biographie/inventai.php (consulté le 4 avril 2014).

8. Respectivement Charles de Mazade, Revue des Deux Mondes, $1^{\mathrm{er}}$ mai 1857, en ligne : http:// flaubert.univ-rouen.fr/etudes/madame_bovary/mb_maz.php (consulté le 4 avril 2014), et SaintRené Taillandier, Revue des Deux Mondes, 15 février 1863, en ligne : http://flaubert.univ-rouen.fr/ etudes/salammbo/sal_sai5.php (consulté le 4 avril 2014).

9. John Greene, "Structure et épistémologie ", dans Flaubert et le "comble de l'art": nouvelles recherches sur Bouvard et Pécuchet, actes du colloque tenu au Collège de France, les 22 et 23 mars 1980, Paris, CDU-SEDES, 1981, p. 113.

10. Raymond Queneau, "Préface à Bouvard et Pécuchet ", dans Bâtons, chiffres et lettres, Paris, Gallimard, 1965 [1950], p. 97-124, ici p. 121, coll. «Idées».

11. Flaubert, Bouvard et Pécuchet, éd. Stéphanie Dord-Crouslé, GF Flammarion, 1999 ( $2^{\mathrm{e} e ́ d .}$ 2008), p. 296.

12. "Le synthétisme est la grande loi de l'ontologie ", lettre à Louis Bouilhet, $1^{\mathrm{er}}$ août 1855 , Corr., op. cit., t. I, p. 252.

13. Laugel, «Les études philosophiques en Angleterre. M. Herbert Spencer, First Principles, by Herbert Spencer, London $1862 »$, op. cit., p. 935.

14. Ibid., p. 934.

15. Herbert Spencer, Classification des sciences, traduit de l'anglais sur la troisième édition par F. Réthoré, Paris, Germer Baillière, 1872, p. 95-136.

16. Ibid., p. 105.

17. Ibid., p. 120.

18. Lettre à Louis Bouilhet, 4 septembre 1850, Corr., op. cit., t. I, p. 679.

19. Ibid., p. 937.

20. Lettre à Marie-Sophie Leroyer de Chantepie, 30 mars 1857, Corr., op. cit., t. II, p. 698.

21. Bouvard et Pécuchet, op. cit., p. 393.

22. Spencer, Premiers principes, op. cit., p. 21 et 24.

23. Laugel, «Les études philosophiques en Angleterre. M. Herbert Spencer, First Principles, by Herbert Spencer, London $1862 »$, op. cit., p. 936.

24. Ibid., p. 955.

25. Pour la phrase citée, lettre à sa nièce Caroline, 23 mars 1868, Corr., op. cit., t. III, p. 738 (c'est Flaubert qui souligne). Les deux autres lettres sont adressées à Edma Roger des Genettes (13 mars 1879, Corr., t. V, p. 579), et à Maxime du Camp (13 novembre 1879, Corr., t. V, p. 740). 
26. Juliette Azoulai, L'Âme et le corps chez Flaubert. Une ontologie simple, thèse soutenue à l'université de Rouen, 16 novembre 2012, sous la direction de Jean-Louis Cabanès et Yvan Leclerc, p. 89, à paraître aux éditions Garnier en 2014.

27. Laugel, «Les études philosophiques en Angleterre. M. Herbert Spencer, First Principles, by Herbert Spencer, London $1862 »$, op. cit., p. 957.

28. Ibid., p. 949.

29. Ottavi, Penser Spencer, op. cit., p. 10.

30. Dossiers de Bouvard et Pécuchet, BM Rouen, ms g 226-8, fo 40 r. En ligne : http://www.dossiersflaubert.fr/cote-g226_8_f_040__r_-_-_ (consulté le 11 mars 2014).

31. Voir les lettres à sa nièce Caroline, [1 ${ }^{\mathrm{er}}$ février 1880], Corr., op. cit., t. V, p. 803 (demande des livres); à Léonie Brainne, [3 février 1880], ibid., p. 817 (Pouchet ne répond pas); à Guy de Maupassant, 13 février 1880, ibid., p. 826 (Pouchet doit envoyer les livres).

32. Dossiers de Bouvard et Pécuchet, ms g 226-2, $\mathrm{f}^{\circ} 168 \mathrm{r}^{\circ}$ et $\mathrm{v}^{\circ}$.

33. Plans et scénarios de Bouvard et Pécuchet, BM Rouen, ms gg 10, fo 18. En ligne: http:// flaubert.univ-rouen.fr/jet/public/trans.php?corpus=pecuchet\&id=6798 (consulté le 11 mars 2014).

34. Voir Yvan Leclerc, La Spirale et le monument, essai sur Bouvard et Pécuchet de Gustave Flaubert, Paris, CDU-SEDES, 1988, p. 124-130.

35. Spencer, De l'éducation intellectuelle, morale et physique, Paris, G. Ballière, 1880, p. 105.

36. Flaubert, Bouvard et Pécuchet, op. cit., p. 377.

37. Ibid., p. 355.

38. Spencer, De l'éducation intellectuelle, morale et physique, op. cit., respectivement p. 47 et 274.

39. «L'exemple sur leurs élèves témoignerait combien la liberté l'emporte sur la contrainte » ( Bouvard et Pécuchet, op. cit., p. 363).

40. Spencer, De l'éducation intellectuelle, morale et physique, op.cit., p.99. Voir dans Bouvard et Pécuchet : «[...] comme la grammaire ne peut être comprise des enfants - et qu'ils la sauront s'ils entendent parler correctement, les deux bonshommes surveillaient leurs discours jusqu'à en être incommodés » (p. 379-380).

41. Spencer, De l'éducation intellectuelle, morale et physique, op. cit., p. 189.

42. Ibid., respectivement p. 155, 96 et 82 . Voir Bouvard et Pécuchet : « Rien n'est stupide comme de faire apprendre par cœur » (p. 356).

43. Flaubert, Bouvard et Pécuchet, op. cit., p. 357-358.

44. Ibid., p. 368.

45. Dossiers de Bouvard et Pécuchet, ms g 225-3, fo $340 \mathrm{v}^{\circ}$.

46. Herbert Spencer, De l'éducation suivi d'Éducation et positivisme, textes choisis et présentés par Dominique Ottavi, Lyon, INRP, 2003, « Présentation », p. XIII.

47. 21 janvier 1880, Corr., op. cit., t. V, p. 791.

48. Flaubert, Bouvard et Pécuchet, op. cit., p. 361.

49. Ibid., p. 379.

50. Ibid., p. 376.

51. Spencer, De l'éducation intellectuelle, morale et physique, op. cit., p. 171.

52. Ibid., p. 176.

53. Flaubert, Bouvard et Pécuchet, op. cit., p. 393.

54. Gisèle Séginger, « Forme romanesque et savoir. Bouvard et Pécuchet et les sciences naturelles », Revue Flaubert, $n^{\circ} 4,2004$. En ligne : http://flaubert.univ-rouen.fr/revue/revue4/02seginger.php (consulté le 11 mars 2014).

55. Respectivement lettres à Louise Colet, 6 avril 1853, Corr., op.cit., t. II, p. 298, et 24 avril 1852, ibid., p. 79.

56. Spencer, De l'éducation intellectuelle, morale et physique, op. cit., p. 73.

57. Ibid., p. 3 et 18. 
58. Ibid., p. 61 et 63.

59. Ibid., p. 15-16 et 63 .

60. Voir l'article de Niklas Bender, «Bouvard et Pécuchet: une mise en scène comique des savoirs ", Arts et Savoirs [En ligne], 1|2012, mis en ligne le 15 février 2012, consulté le 09 novembre 2015. URL : http://lodel.revues.org/10/aes/566.

61. Jeremy Bentham, Théorie des peines et des récompenses, 1818 [1811].

62. Spencer, De l'éducation intellectuelle, morale et physique, op. cit., p. 189.

63. Jean-Jacques Rousseau, CEuvres complètes, nouvelle édition, Paris, 1788-1793, 38 vol. Émile ou de l'éducation, vol. 10-13, ici vol. 10, p. 213.

64. Flaubert, Bouvard et Pécuchet, op. cit., p. 376.

65. Spencer, De l'éducation intellectuelle, morale et physique, op. cit., p. 190 et suiv.

66. Ibid., p. 140.

67. Claude Digeon, Le Dernier Visage de Flaubert, Paris, Aubier, 1946, p. 101.

68. Laugel, «Les études philosophiques en Angleterre. M. Herbert Spencer, First Principles, by Herbert Spencer, London 1862 », op. cit., p. 935.

INDEX

Mots-clés : Flaubert (Gustave), Bouvard et Pécuchet, littérature, philosophie, correspondance

\section{AUTEUR}

\section{YVAN LECLERC}

Professeur à l'université de Rouen, directeur du Centre Flaubert, laboratoire CÉRÉdI 